diagnosed as CD, whose initial diagnostic workup suggested TB.

Methods A 23 year young female presented in 2013 with complaints of abdominal pain of one year duration and weight loss of $5 \mathrm{~kg}$ and anorexia since one month. She was a known case of $\beta$ thalassemia minor. General and abdominal examination were unremarkable. Investigations revealed microcytic hypochromic anaemia, ESR- 14, normal albumin and chest roentgenogram. Colonoscopy was suggestive of ileocaecal valve deformity with ulcers and terminal ileal ulcers and normal colonic mucosa. Histopathological examination of ileal biopsy was reported as severe typhilitis, and mycobacterial DNA PCR of ileal biopsy had detected M. Tuberculosis complex. She was started on four drugs first line antitubercular therapy in 2013 for ileocaecal tuberculosis. At 4 months follow up, abdominal pain was present but with reduced frequency and she had gained $3 \mathrm{~kg}$ weight. She again came for follow up in 2014 after taking antitubercular therapy for 9 months with complaints of abdominal pain and significant weight loss. She had undergone colonoscopy and laparoscopy at our centre for the diagnostic dilemma of tuberculosis/ Crohn's disease.

Results Colonoscopy had revealed oedematous ileocaecal valve with terminal ileum nodularity and ulceration. Laparoscopy had revealed creeping fat and a diseased terminal ileal segment with mesenteric lymphadenopathy without evidence of omental thickening or peritoneal nodularity. Histopathology of terminal ileum had revealed moderate ileitis with non-caseating epitheloid cell granuloma without giant cell. She was started on immunosuppressive therapy for Crohn's disease and responded to treatment. She was in remission after 3 years of follow up.

Conclusions Distinguishing between $\mathrm{TB}$ and $\mathrm{CD}$ is difficult because of varied clinical features, similar pathological, imaging and endoscopic appearances. This case represents CD misdiagnosed as TB in a young lady as tissue TB PCR was false positive and it has low sensitivity than previously described.

\section{IDDF2018-ABS-0044 ENTERIC PATHOGENS AND PREDICTORS FOR ACUTE DIARRHOEA IN CHILDREN LIVING WITH HUMAN IMMUNODEFICIENCY VIRUS INFECTION}

${ }^{1}$ Pooja Dewan*, 'Dhano Mardi, 'Sunil Gomber, ${ }^{2}$ Rumpa Saha. ${ }^{1}$ Department of Pediatrics, University College of Medical Sciences, Delhi, India; ${ }^{2}$ Department Of Microbiology, University College of Medical Sciences, Delhi, India

\subsection{6/gutinl-2018-IDDFabstracts.80}

Background Understanding the aetiology of diarrhoea in Human immunodeficiency virus (HIV) infected children and its predictors can help reduce mortality in this vulnerable group.

Aims

1. To compare the prevalence of enteric pathogens in HIVinfected children with acute diarrhoea and without diarrhoea

2. To assess the association between carriage of enteric pathogens in HIV-infected children and the aetiology/

frequency of diarrheal episodes within the next 3 months.

Methods We evaluated HIV-infected children with acute diarrhoea (cases) and without diarrhoea (controls) aged 18 months
-12 years attending the anti-retroviral clinic at a tertiary hospital in Delhi. Children who had received any antimicrobial therapy within the previous 2 weeks were excluded. A single stool sample was collected for microscopic examination, bacterial culture and microscopic examination including modified acid-fast staining (oocysts of Cryptosporidium, Isospora and Cyclospora) and Trichrome 1staining (for oocysts of Microsporidia). Serology for Cryptosporidium parvum was determined. All children were followed up for three months for the occurrence of diarrhoea.

Results Enteric pathogens were isolated in $48.8 \%$ cases $(n=41)$ and $42 \%$ controls $(n=52)$. The common pathogens isolated in the diarrheal and non-diarrheal groups were Cryptosporidium $(29.3 \%$ Vs $17.3 \%)$, E. coli $(29.3 \%$ Vs $17.3 \%)$, Giardia $(14.6 \%$ Vs $5.8 \%)$, and Yeast $(4.8 \%$ Vs 0$)$; $(\mathrm{p}<0.05)$. During follow up, 8 cases (19.1\%) and 8 controls (15.3\%) had a diarrheal recurrence. The pathogen isolated in subsequent episodes matched with the isolate in 3 controls and 3 cases. Severe thinning $(\mathrm{BMIZ}<-3)$, severe underweight $(\mathrm{WAZ}<-3)$, and severe immunodeficiency (CD4 <15\%) were significant predictors for acute diarrhoea. Co-trimoxazole prophylaxis did not offer any significant protection from acute diarrhoea but prevented infection with Microsporidia and Isospora.

Conclusions Cryptosporidium parvum is harboured commonly in asymptomatic HIV-infected children and predisposes to future diarrheal occurrence in them. A longer duration of treatment for acute diarrheal episodes may be needed in HIVinfected children. Children with severe thinning, underweight and immunodeficient must be screened for the presence of enteric pathogens and treated pre-emptively.

\section{IDDF2018-ABS-0045 INCIDENTAL GALL BLADDER CANCER IN LAPAROSCOPIC CHOLECYSTECTOMY}

Tripuraneni Venkata Aditya Chowdary*, Ramalingam Trivikraman. Gleneagles Global Hospital, India

\subsection{6/gutjnl-2018-IDDFabstracts.81}

Background Carcinoma of the Gall Bladder (GBC) is the most common malignancy of the biliary tract and sixth most common gastrointesnal malignancy worldwide. Laparoscopic cholecystectomy has now become the most commonly performed major surgery worldwide, and an increasing number of pathological specimens are said to be showing incidental malignancy. The overall incidence is around $0.2 \%-2.9 \%$.

Methods A retrospective study was done by reviewing records of patients who underwent Laparoscopic cholecystectomy at our centre between 2012-2016 (5 years). A total of 2758 cases were included in the study. Their mode of presentation, duration of symptoms, pre-operative imaging, intraoperative findings and histopathological reports were analysed.

Results A total of 6 cases of incidental GBC were identified. The overall incidence was around $0.2 \%$. The mean age of the group was 60 years of which 4 were females and 2 were males. On pathological analysis, one patient had a background of acute cholecystitis, one had chronic cholecystitis, one had a polyp, and the remaining had unremarkable histology in the remainder of the gallbladder. The staging was T2N1M0 in two patients, T1bNM0 in two patients and T3N1M0 in two 